\title{
CONCENTRATION-CANCELLATION AND HARDY SPACES
}

\author{
ITALO VECCHI \\ (Received 21 August 1991; revised 10 March 1992) \\ Communicated by P. E. Kloeden
}

\begin{abstract}
Let $v^{\epsilon}$ be a sequence of DiPerna-Majda approximate solutions to the 2-d incompressible Euler equations. We prove that if the vorticity sequence is weakly compact in the Hardy space $H^{1}\left(R^{2}\right)$ then a subsequence of $v^{\epsilon}$ converges strongly in the energy norm to a solution of the Euler equations.
\end{abstract}

1992 Mathematics subject classification (Amer. Math. Soc.): primary 35Q10; secondary 76D05.

The convergence of approximate solutions $v^{\epsilon}$ of the 2-d inviscid Euler equations as the regularization parameter $\epsilon$ goes to zero has been studied by DiPerna and Majda in a series of papers $([4,5,6])$. In $[4,5]$ they give several examples of sequences of compactly supported approximate solutions $v^{\epsilon}$ (as defined in [4, Definition 1.1]) whose vorticity $\omega^{\epsilon}$ is bounded in $L^{1}$ which fail to be compact in $L^{2}$ so that in the limit concentration phenomena occur. Moreover in [4, Theorem 1.3] a criterion which rules concentrations out is proposed: it is shown that a uniform bound on a logarithmic Morrey norm of $\omega^{\epsilon}$ yields strong $L^{2}$-convergence of the velocity field.

In this note a different criterion for compactness is introduced: we show that strong $L^{2}$-compactness of $v^{\epsilon}$ follows from weak compactness of $\omega^{\epsilon}$ in the Hardy space $H^{1}\left(R^{2}\right)$. Since $H^{1}\left(R^{2}\right)$ is not rearrangement invariant the fine structure of the vorticity plays a crucial role in getting strong $L^{2}$-convergence. We recall that by the Dunford-Pettis theorem (see [7, VIII, Theorem 1.3]) a necessary and sufficient condition for a subset $\Lambda$ of $L^{1}\left(R^{2}\right)$ to be weakly pre-compact in $L^{1}(\Omega)$ is that

$$
\lim _{s \rightarrow+\infty}\left(\sup _{f \in \Lambda} \int_{|x|>s}|f| d x\right)=0
$$

(C) 1995 Australian Mathematical Society 0263-6115/95 \$A2.00+0.00 
and that there exists a positive function $G(s): R^{+} \rightarrow R^{+}$such that

$$
\lim _{s \rightarrow+\infty} G(s) / s=+\infty
$$

and

$$
\sup _{f \in \Lambda} \int_{R^{2}} G(|f|) d x<+\infty .
$$

Let $R_{i}, i=1,2$, denote the Riesz transforms:

$$
R_{j} f(x)=\int_{R^{2}} \frac{x_{j}-y_{j}}{|x-y|^{3}} f(y) d x .
$$

We adopt both notation and terminology of [4]. We formulate our result as follows.

THEOREM 1. Let $v^{\epsilon}$ be a sequence of approximate solutions such that $\omega^{\epsilon} \in H^{1}\left(R^{2}\right)$ $\cap C_{o}^{\infty}\left(R^{2}\right)$ and that for $t \geq 0$

$$
\left\|\omega^{\epsilon}(., t)\right\|_{H^{\prime}}<C, \quad 0<\epsilon \leq \epsilon_{0} .
$$

Moreover let there be a function $G(s): R^{+} \rightarrow R^{+}$such that (1) and (2) hold for $\Lambda=\left\{\omega^{\epsilon}\right\},\left\{R_{i} \omega^{\epsilon}\right\}, i=1,2$.

Then there is a subsequence of $v^{\epsilon}$ which converges strongly in $L_{l o c}^{2}$ to a weak classical solution $v$ of the Euler equations. Moreover $v \in W^{1,1}\left(R^{2}\right)$.

We recall (see [8, Ch. XIV]) that a function $f$ belongs to the Hardy space $H^{1}\left(R^{2}\right)$ if and only if there is a sequence of numbers $\lambda_{j}$ satisfying $\sum_{1}^{\infty}\left|\lambda_{j}\right|<\infty$ and a series of functions (atoms) $a_{j}$ such that

$$
f=\sum_{1}^{\infty} \lambda_{j} a_{j}
$$

where the $a_{j}^{\prime} s$ have the following properties:

(a) $a_{j}$ is supported on a ball $B_{j}$ and $\left\|a_{j}\right\|_{\infty}<1 /\left|B_{j}\right|$;

(b) $\int_{R^{2}} a_{j}(x) d x=0$.

The $H^{1}$-norm of $f$ can be defined as the infimum of the expressions $\sum_{1}^{\infty}\left|\lambda_{j}\right|$ on all possible representations of $f$ as in (4).

If Condition (b) were dropped the resulting space would be $L^{1}\left(R^{2}\right)$. The subtle cancellation effect due to (b) (cf. 'phantom vortices' in [4, 1.A]) is crucial in obtaining strong $L^{2}$-compactness.

PROOF OF THEOREM 1. To prove the theorem we introduce the stream function $\psi^{\epsilon}$ such that

$$
\Delta \psi^{\epsilon}=\omega^{\epsilon}
$$


and we proceed as in the proof of [4, Theorem 3.1]. It is known (see [8, Chapter XV]) that for every $f$ in $B M O\left(R^{2}\right)$ there are $g_{i}$ in $L^{\infty}\left(R^{2}\right), i=0,1,2$, such that

$$
f=g_{0}+\sum_{i=1,2} R_{i} g_{i}
$$

Hence

$$
\int_{R^{2}} f \omega^{\epsilon} d x=\int_{R^{2}} \omega^{\epsilon}\left(g_{0}+\sum_{i=1,2} R_{i} g_{i}\right) d x=\int_{R^{2}} \omega^{\epsilon} g_{0}-\sum_{i=1,2} g_{i} R_{i} \omega^{\epsilon} d x
$$

By our Assumption (2) the sequence $\left\{\omega^{\epsilon}\right\}$ and its Riesz transforms admit a weakly convergent subsequence in $L^{1}\left(R^{2}\right)$. Therefore there is a subsequence such that

$$
\omega^{\epsilon} \rightarrow \omega \quad \text { weakly in } H^{\mathrm{l}}\left(R^{2}\right) \text {. }
$$

The statement of Theorem 1 is guaranteed (see [4, (3.6)]) by showing that for all $\rho \in C_{o}^{\infty}\left(R^{2}\right)$

$$
\lim _{\epsilon \rightarrow 0} \int_{R^{2}} \rho\left|v^{\epsilon}\right|^{2} d x=\int_{R^{2}} \rho|v|^{2} d x .
$$

Indeed after integrating by parts (7) is seen to hold if and only if (see [4, (3.7)-(3.10)])

$$
\lim _{\epsilon \rightarrow 0} \int_{R^{2}} \rho \psi^{\epsilon} \omega^{\epsilon} d x=\int_{R^{2}} \rho \psi d \omega
$$

where $\psi$ is the stream function corresponding to $\omega$ in (5).

We recall that

$$
\frac{\partial^{2}}{\partial x_{j} \partial x_{k}} f=-R_{j} R_{k} \Delta f
$$

Hence

$$
\frac{\partial^{2}}{\partial x_{j} \partial x_{k}} \psi^{\epsilon}=-R_{j} R_{k} \omega^{\epsilon}
$$

Since the Riesz transform maps $H^{1}\left(R^{2}\right)$ continuously into itself we obtain

$$
\left\|\frac{\partial^{2}}{\partial x_{j} \partial x_{k}} \psi^{\epsilon}\right\|_{L^{1}} \leq\left\|\frac{\partial^{2}}{\partial x_{j} \partial x_{k}} \psi^{\epsilon}\right\|_{H^{1}} \leq C\left\|\omega^{\epsilon}\right\|_{H^{1}}
$$

and $\psi^{\epsilon}$ remains bounded in $W^{2,1}\left(R^{2}\right)$.

We recall that for any bounded domain $\Omega$ in $R^{2}$, by the Gagliardo-Sobolev imbedding theorem, $W^{2,1}\left(R^{2}\right)$ is continuously imbedded in $C(\bar{\Omega})$.

Therefore by (9),

$$
\left\|\psi^{\epsilon}\right\|_{C(\bar{\Omega})} \leq C\left\|\omega^{\epsilon}\right\|_{H^{\prime}}
$$


Moreover (see [1, Lemma 5.8]) if $u \in W^{2,1}\left(R^{2}\right)$ for any $P_{o} \in R^{2}$ we have that for $\delta>0$, and $|\Delta P|<\delta / 2$

(11) $\left|u\left(P_{o}+\Delta P\right)-u\left(P_{o}\right)\right| \leq C\left(\frac{1}{\delta^{2}}\|u(P+\Delta P)-u(P)\|_{L^{1}\left(B_{\delta}\left(P_{o}\right)\right)}\right.$

$$
\begin{aligned}
& +\frac{1}{\delta} \sum_{i}\left\|\frac{\partial}{\partial x_{i}} u(P+\Delta P)-\frac{\partial}{\partial x_{i}} u(P)\right\|_{L^{1}\left(B_{\delta}\left(P_{o}\right)\right)} \\
& \left.+\sum_{i, j}\left\|\frac{\partial^{2}}{\partial x_{j} \partial x_{i}} u(P+\Delta P)-\frac{\partial^{2}}{\partial x_{j} \partial x_{i}} u(P)\right\|_{L^{1}\left(B_{\delta}\left(P_{o}\right)\right)}\right) .
\end{aligned}
$$

By (weak) continuity of the Riesz transforms from $H^{1}\left(R^{2}\right)$ into itself there is a subsequence of $\partial^{2} \psi^{\epsilon} / \partial x_{j} \partial x_{k}$ that converges weakly in $H^{1}\left(R^{2}\right)$ to a $\phi_{i, j} \in H^{1}\left(R^{2}\right)$. On the other hand weak convergence in $H^{1}\left(R^{2}\right)$ implies weak convergence in $L^{1}(\Omega)$ (indeed $L^{\infty} \subset B M O$ ) so that we have

$$
\frac{\partial^{2}}{\partial x_{j} \partial x_{k}} \psi^{\epsilon} \rightarrow \phi_{i, j} \quad \text { weakly in } L^{1}\left(R^{2}\right) .
$$

By the full version of the Dunford-Pettis Theorem, for every $\kappa>0$ there is a $\delta>0$ such that for any $P \in \Omega$

$$
\left\|\frac{\partial^{2}}{\partial x_{j} \partial x_{k}} \psi^{\epsilon}\right\|_{L^{\prime}\left(B_{\delta}(P)\right)}<\kappa
$$

uniformly in $\epsilon$.

We observe that if $|\Delta P|<\delta$,

$$
\left\|\frac{\partial^{2}}{\partial x_{j} \partial x_{k}}\left[\psi^{\epsilon}(P+\Delta P)-\psi^{\epsilon}(P)\right]\right\|_{L^{1}\left(B_{\delta}\left(P_{o}\right)\right)}<C\left\|\frac{\partial^{2}}{\partial x_{j} \partial x_{k}} \psi^{\epsilon}\right\|_{L^{1}\left(B_{2 \delta}\left(P_{o}\right)\right)} .
$$

Therefore for every $P_{o}$, given $\kappa_{o}>0$ we can find a $\delta_{o}>0$ such that if $|\Delta P|<\delta_{o} / 2$

$$
\sum_{j, k}\left\|\frac{\partial^{2}}{\partial x_{j} \partial x_{k}}\left[\psi^{\epsilon}(P+\Delta P)-\psi^{\epsilon}(P)\right]\right\|_{L^{1}\left(B_{\delta_{o}}\left(P_{o}\right)\right)}<\frac{\kappa_{o}}{3}
$$

uniformly in $\epsilon$. Moreover since $W^{1,1}(\Omega)$ is compactly imbedded in $L^{p}$ for any $p<2$, by (9) both $\left\{\psi^{\epsilon}\right\}$ and $\left\{\partial \psi^{\epsilon} / \partial x_{i}\right\}$ are compact in $L^{1}$. Hence by the Kondratchev compactness criterion (see [1]) there is a $\delta_{1}>0$ such that if $|\Delta P|<\delta_{1}$,

$$
\begin{aligned}
\frac{1}{\delta_{o}^{2}}\|u(P+\Delta P)-u(P)\|_{L^{1}\left(B_{\delta_{o}}\left(P_{o}\right)\right)} & <\frac{\kappa_{o}}{3}, \\
\frac{1}{\delta_{o}} \sum_{i}\left\|\frac{\partial}{\partial x_{i}} u(P+\Delta P)-\frac{\partial}{\partial x_{i}} u(P)\right\|_{L^{1}\left(B_{\delta}\left(P_{o}\right)\right)} & <\frac{\kappa_{o}}{3},
\end{aligned}
$$


and by (11)

$$
\left|\psi^{\epsilon}\left(P_{o}+\Delta P\right)-u\left(P_{o}\right)\right|<\kappa_{o}
$$

uniformly in $\epsilon$. The sequence $\psi^{\epsilon}$ is equicontinuous by (12) and by Ascoli's theorem we can extract a subsequence such that

$$
\psi^{\epsilon} \rightarrow \psi \quad \text { strongly in } C(\Omega)
$$

By (6) we have that $\omega^{\epsilon} \rightarrow \omega$ weakly in the space of Radon measures $M(\Omega)$ so that (8) holds and the same argument as in [4, Theorem 1.3] yields the statement of the theorem.

REMARK. The first example in $[4,(1, \S A)]$ (phantom vortices) shows a sequence of vorticities which stays bounded in $H^{1}\left(R^{2}\right)$ whose velocity field fails to converge strongly in $L^{2}$; in the second example one has strong $L^{1}(\Omega)$ convergence of the vorticity but the sequence does not lie in $H^{1}\left(R^{2}\right)$ and again concentrations occur. By looking at the proof of Delort's recent deep result [3] a condition weaker than weak convergence of $\omega^{\epsilon}$ in $L^{1}(\Omega)$ is sufficient to pass to the limit in the quadratic terms of the Euler equations, due to their special structure, although concentrations may occur. It is interesting that every bounded sequence in $H^{1}\left(R^{2}\right)$ admits a weakly (*) convergent subsequence whose limit stays in $H^{1}\left(R^{2}\right)$ (see [2, Lemma (4.2)]). However, since $(V M O)^{*}=H^{1}\left(R^{2}\right)$ and $L^{\infty} \not \subset V M O$, this does not yield weak $L^{1}$-convergence.

It is worth observing that Condition (2) for $\omega^{\epsilon}$ is rearrangement invariant and so in the time dependent case it is conserved by the particle trajectory map. On the other hand, as for the bounds of [4, Theorem 3.1, (3.4)], it is not clear what happens to the $H^{1}$-norm as time goes by, since $H^{1}\left(R^{2}\right)$ is not rearrangement invariant. It would be interesting to have a less cumbersome characterization of weak compactness in $H^{1}$ than the one given here.

\section{References}

[1] R. Adams, Sobolev spaces (Academic Press, New York, 1975).

[2] R. R. Coifman and G. Weiss, 'Extensions of Hardy spaces', Bull. Amer. Math. Soc. (N.S.) 83 (1977), $569-645$.

[3] J. Delort, 'Existence de nappes de tourbillon en dimension deux', J. Amer. Math. Soc. 4 (1991), 553-586.

[4] R. J. DiPerna and A. Majda, 'Concentrations in regularizations for 2-D incompressible flow', Comm. Pure Appl. Math. 40 (1987), 302-345.

[5] — ' 'Oscillations and concentrations in weak solutions of the incompressible fluid equations', Comm. Math. Phys. 108 (1987), 667-689.

[6] — - 'Reduced Hausdorff dimension and concentration cancellation for 2-D incompressible flow', J. Amer. Math. Soc. 1 (1988), 59-95. 
[7] I. Ekeland and R. Temam, Convex analysis and variational problems (North Holland, Amsterdam, 1982).

[8] A. Torchinsky, Real variable methods in harmonic analysis (Academic Press, New York, 1986).

Seminar für Angewandte Mathematik

ETH-Zentrum

CH-8092, Zürich

Switzerland 